Asian Journal of Engineering and Applied Technology

ISSN 2249-068X Vol. 7 No. S2, 2018, pp.6-11

(C) The Research Publication, www.trp.org.in

\title{
Feature Based Comparison of Text Based Image Retrieval and Context Based Image Retrieval Images
}

\author{
Gagandeep Kaur $^{1}$, Deepam Goyal ${ }^{2}$ and Rajeev Kumar Dang ${ }^{3}$ \\ ${ }^{1}$ Department of Computer Science, Guru Nanak College,Budhlada, Punjab, India \\ ${ }^{2}$ Department of Mechanical Engineering, National institute of Technical Teachers Training \& Research, Chandigarh, India \\ ${ }^{3}$ Department of Mechanical Engineering, SSGPURC, Hoshiarpur, Punjab, India \\ E-Mail: dang.rajeev@pu.ac.in
}

\begin{abstract}
Image processing is a field to process the images according to horizontal and vertical axis to form some useful results. It deals with edge detection, image compression, noise removal, image segmentation, image identification, image retrieval and image variation etc. Customarily, there are two techniques i.e. text based image retrieval and content based image retrieval that are used for retrieving the image according to features and providing color to all pixel pairs. The system retrieval that is based on TBIR assists to recover an image from the database using annotations. CBIR extorts images to form a hefty degree database using the visual contents of an original image that is called low level features or features of an image. These visual features are extracted using feature extraction and then match with the input image. Histogram, color moment, color correlogram, Gabor filter and wavelet transform are various CBIR techniques that can be used autonomously or pooled to acquire enhanced consequences. This paper states about a novel technique for fetching the images from the image database using two low level features namely color based feature and texture based features. Two techniques- one is color correlogram (for color indexing) and another is wavelet transform (for texture processing) has also been introduced.

Keywords:Context based image retrieval (CBIR), Feature extraction; Text based image retrieval (TBIR)
\end{abstract}

\section{INTRODUCTION}

Advancement in technology is also amplified the digital image, videos and other media files. As the upshot of digital media, the dimension of these files is also increased. This scattering out in the digital image required able-bodied defined methods for conduct them i.e. penetrating, scrutinizing, and sorting right through outsized image database [1].Entire the media files images are most prominentaspect.Information retrieval is the field of acquaintance that compact with the demonstration,storage,andadmittanceofinformationalobjects .Additionaldistinctively, while the repossessed information is a variety of images, this fieldof acquaintance is known as Image Retrieval. These techniques are based on text explanation of an images[2,3]. However this entirely textbased approach pretense two noteworthy boundaries in the reclamation of images. The first limitation was connected with the dimensions of the database. Manual annotation was such a burdensome and posh task that it could not be functional to outsized image databases. The second limitation which exaggerated the concert of the system was that the explanation of the images was establish to be an extremely slanted task that could engender different text labels to the similar image. Image indexing techniques solves some Problems associated with contents like color, texture and shape[4].

\section{RELATED WORK}

Sirisha et al.,[5]developed an application for medical field . In this system color correlogram, color mean and wavelet decomposition is used for retrieving the images from the database. Support Vector Machine (SVM) classifier is used for the testing final feature of input image with the stored images[6]. Haridas et al.,[7]proposed an approach for CBIR using RGB color histogram, tamura texture and gabor features. Testing and implementation methods are based on these three features i.e. precision value, recall value and accuracy rate[8]. CBIR Systems are implemented with the help of java[9]. Deole et al.,[10] developed a new retrieval technique that is based on contents and uses some color features of an image with k-nearest algorithm for classification of an image[11,12]. k-nearest neighbor calculations calculates the difference between the training vector and the test vector[13]. Jain [14]presented two contents of an image color and texture for retrieving the image from the database. Histograms are used to give different color and Gabor filter are used to give texture to the image. Gabor filter is a tool for texture feature extraction[15]. Bala et al.,[16] introduced a new technique for multiple features. To improve the system performance this technique uses the feature of fuzzy logic. Fuzzy logic is used to solve the semantic gap. Fuzzy logic computes the attributes and features of the image using the rules. Multiple features of an image are used in this proposed technique because Image contents are not exemplified accurately with one feature, but better results are formed with all features combinations[17].

\section{A. Textbased image retrieval}

This technique accepts some keywords, phrases or comments as input query from the user. These annotations and keywords are hand out along with the images separately 
when they stored in database[18].Input query keywords are matched with the keywords that are hand out with images in the database and matching images are processed as output results. No features are extracted from the im- ages. All processing is based on the annotations. For image indexing metadata is used. Theory that works behind the text-based image retrieval system is used to generate a relation between the keywords or comments or input text with the title of an image[19].

\section{B. Context based image retrieval}

It is a system that does not require such keywords or annotations. CBIR system was introduced in 90's to reduce all the limitations of the text based retrieval system of an images[20,21]. CBIR is provoked from the speedy escalation of the digital media that is also increasing the size of the database. This system requires rapidly and fine retrieval of images from such huge storage (database) throughout any condition[6].

\section{III.COLOR CORRELOGRAM}

As we know image is a combination of pixels. Division of pixels is done with the help of color correlogram[22]. To provide different colors to all pairs, spatial correlation is used. When color distribution histogram is compared with the correlogram then it gives measurable values of close modules that gives better outcomes from retrieval point of view, but this is more expensive due to high mathematically coordinates[13].

A color correlogram is a table in the form of rows and columns indexed by color pairs, where the probability of finding a pixel of color $m$ at a distance $n$ from a pixel of color $l$ in the image is specified by the $n$th entry for $(l$, $m)$ [17]. Color correlogram can be defined as:

$\gamma(d)_{(l, m)}(I)=\operatorname{Pr}_{p 1 \epsilon I(l)}\left[p 2 \epsilon I_{c(m)}|p 1-p 2|=n\right]$

where, $\gamma(d)_{(l, m)}(I)$ is color correlogram, $(l, m)$ are color pairs, $p 1$ and $p 2$ are pixels, nis distance between pixels. The distance between pixels $p 1$ and $p 2$ is represented by $\mid \mathrm{p} 1$ $\mathrm{p} 2 \mid$ and $(l, m) \epsilon 1,2, \ldots \mathrm{N}, \mathrm{n} \in\{1,2, \ldots, \mathrm{d}\}$.

If we add the all possible combinations of colors then size of correlogram will be very increases at a great extent. To decrease the size and simplification of the equations of features we always used color autocorrelogram[23].Color auto-correlogram is used to find the spatial correspondence between similar type of colors and reduces the dimension to $\mathrm{O}(\mathrm{Nd})[17]$.

\section{A.Transform ' $T$ ' wavelet pyramid}

T-Wavelets of particular image is considered it gives $\mathrm{T}$ Wavelet Pyramid at different levels. First Wavelet is applied on $\mathrm{x}$ axis (horizontal) and $\mathrm{y}$ axis(vertical) images and diagonals[17]. After the first level Haar Wavelet is applied on first phase. The T-Wavelet pyramid of an image is shown in Figure1. In an image, there are three sections where the T-Wavelet pyramid is applied. The levels of TWavelet are level-1, T-Wavelet level-2 and T-Wavelets level-3.

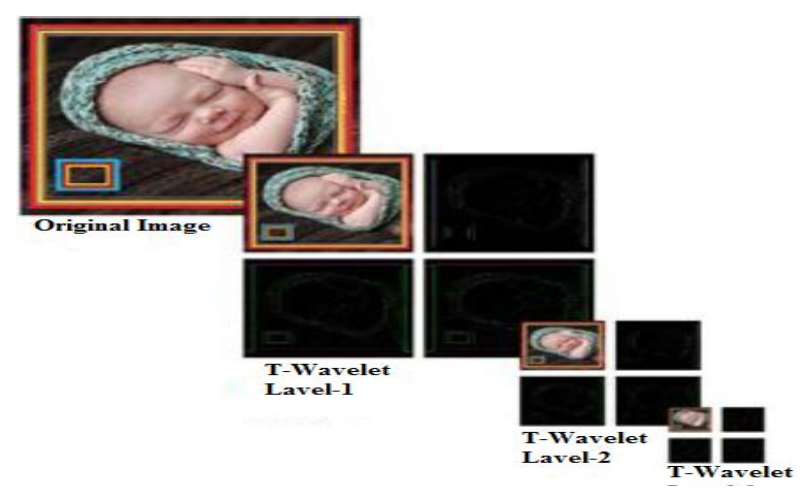

Fig. 1Three Levels of T-Wavelet Pyramid [4]

\section{B. Steps used in energy level algorithm}

The various steps used for decomposing the image are as follows:

1. Decompose an Image in to three levels.

2. Calculate energy at each level using the below equation [6].

$$
E=\frac{1}{X Y} \sum_{i=1}^{x} \sum_{j=1}^{y}|I(i, j)|
$$

3. Here $X$-axis and $Y$-axis represented by $X$ and $Y$ dimensions of the image. The intensity of the pixel located at ith row and jth column of the image map is denoted by $I$.

4. Repeat the first step for sub-bands until the index will be equal to 3 .

5. Use this algorithm for sub-bands and calculate energy at all levels and images are divided into sub band levels. At each level values are stored with the help of Euclidean Distance [19].

\section{Euclidean distance algorithm steps}

1. Fragment the image ' $i$ ' into subparts.

2. Get the energies of the all levels using energy equation

3. Calculate the $k$-energies for all levels of the concerned image $i$ in the database.

4. By applying distance formula, calculate Euclidean distance between the two segments of varying energies

$$
D_{i}=\sum_{k=1}^{k}\left(x_{k}-y_{i, k}\right)^{2}(3)
$$

5. Increment $\mathrm{i}$ all the times. Repeat from step 3.

Every time when distance is calculated between two energy levels values are stored at each extent[24].After finding distance between all energy levels an array of Euclidean distances is sorted. Euclidean distance is calculated between two pints. An image is combination of pixels. There are too many pixels in an image, two points i.e. starting point and ending point have been selected in this work. Afterwards, the difference between two pixels were found. Euclidean distance has been calculated as $\sqrt{ }\left(\mathrm{x}_{2}-\mathrm{x}_{1}\right)^{2}+\left(\mathrm{y}_{2}-\mathrm{y}_{1}\right)^{2}$ as shown in Fig. 3 


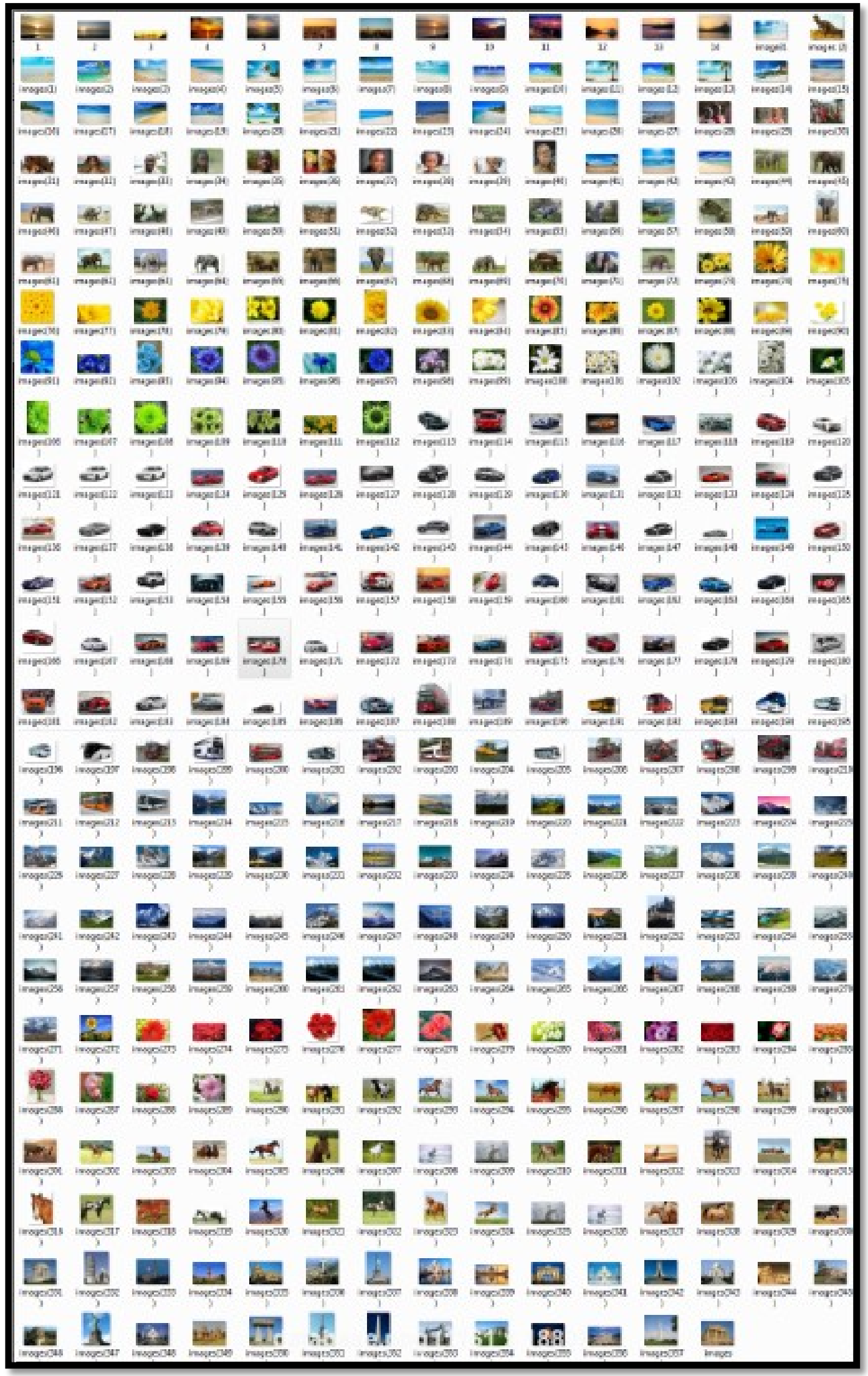

Fig. 2Image Database 


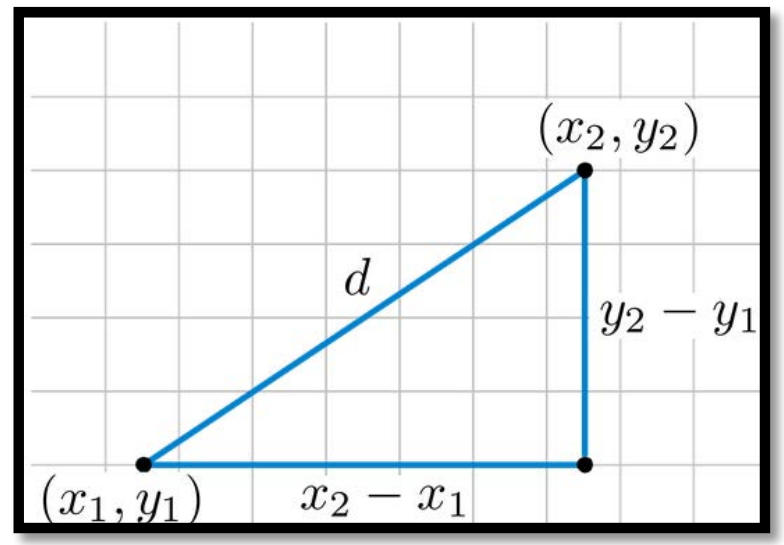

Fig. 3 Euclidean distance

\section{IV.RESULTS}

The image database that we are using in our paper contains 373 8-bit uncompressed bit maps JPGs that have been randomly selected from the World Wide Web. Figure 2 depicts a sample of images in the database. There is large collection of images in database then we randomly select the images from data base and apply $\mathrm{T}$ wavelet. After first step $\mathrm{T}$ wavelet shows similar images that are related to first image which is given as input. You can select any type of images like jpeg, PNG etc. Figure 3 shows how to calculate difference between two points using Euclidean distance formula.

After obtaining all the necessary terms for a number of images in our database, the results were implemented in the final equation. The three images were selected from the database as shown in Figure 4.
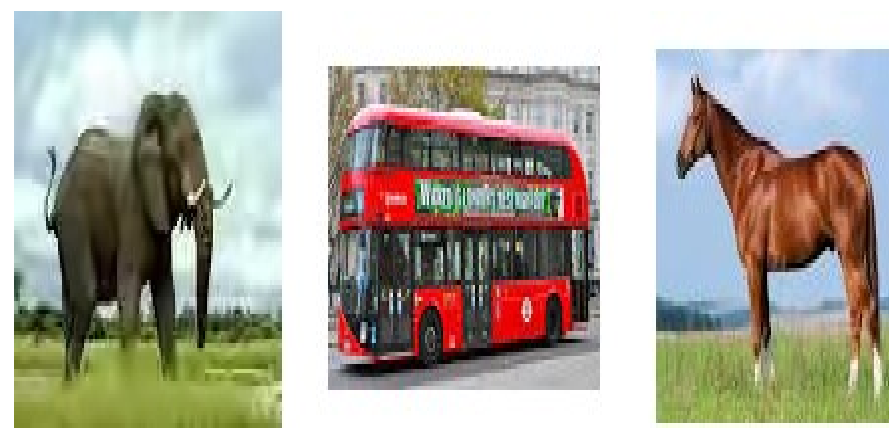

Fig. 4 Tested image

Then, T-Wavelet algorithm was applied on first image of elephant. It gives $\mathrm{T}$ wavelet of an image at different levels similar images are fetched from database automatically. Energy was calculated using equation at different level of this image. Now, the color distance between the original images that are stored in database and tested images that are shown in results were calculated. After this, Euclidean distance was calculated from the varying segments of energies. Figure 5 shows the results of elephant image after applying wavelet at different levels.

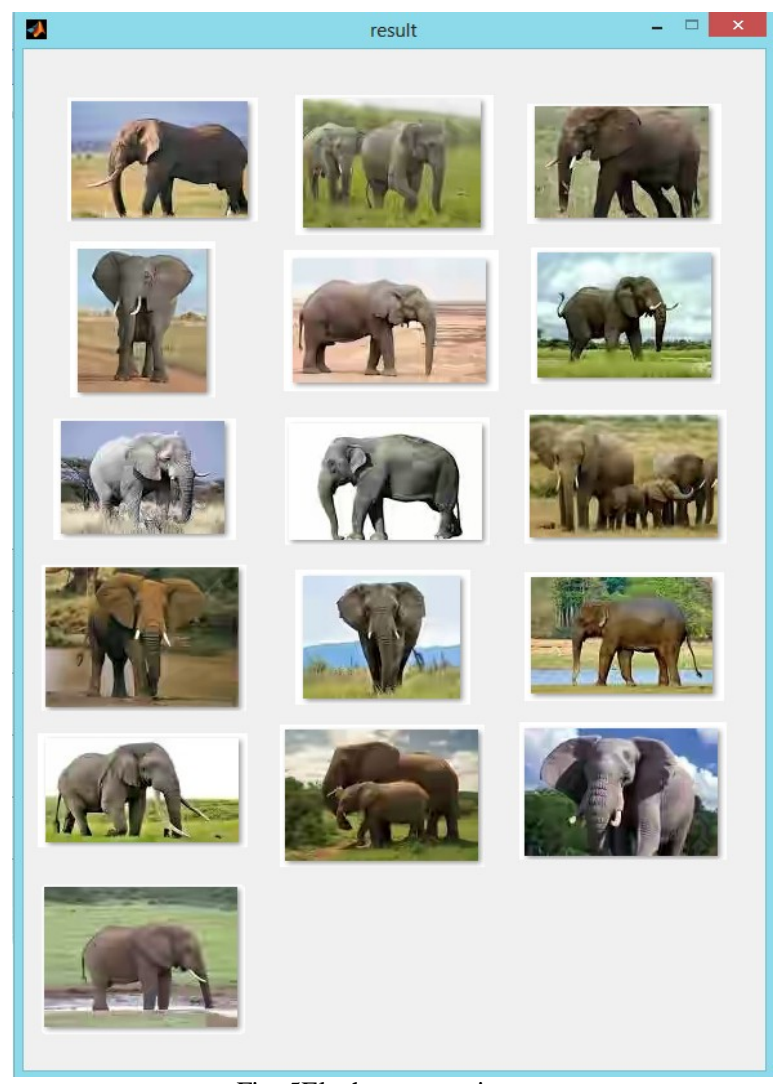

Fig. 5Elephant query image

TABLE I COLOR AND EUCLIDEAN DISTANCE BETWEEN QUERY IMAGE AND RESULTS

\begin{tabular}{|c|c|c|}
\hline File name & Color distance & Euclidean distance \\
\hline Images(62).jpg & 0 & 0 \\
\hline Images(46).jpg & 2.1401 & 1.1449 \\
\hline Images(60).jpg & 3.3804 & 2.4609 \\
\hline Images(45).jpg & 4.3435 & 2.6926 \\
\hline Images(44).jpg & 5.08 & 2.767 \\
\hline Images(67).jpg & 5.765 & 3 \\
\hline Images(64).jpg & 5.879 & 3 \\
\hline Images(61).jpg & 5.994 & 3.23 \\
\hline Images(65).jpg & 6.61 & 3 \\
\hline Images(66).jpg & 6.9638 & 3.786 \\
\hline Images(69).jpg & 7.0813 & 3.84 \\
\hline Images(63).jpg & 7.106 & 3.975 \\
\hline Images(68).jpg & 7.221 & 4.012 \\
\hline Images(70).jpg & 7.7456 & 4.458 \\
\hline Images(72).jpg & 7.845 & 4.6802 \\
\hline Images(71).jpg & 8.1451 & 4.8254 \\
\hline
\end{tabular}

One by one three images like elephant image, Bus image and Horse image that are selected from database, these shows similar images according to their input after applying $\mathrm{T}$ wavelet. Table 1 shows the color distance Euclidean distance values of different images on which we applied $\mathrm{T}$ wavelet. Color distance is calculated using quadratic 
equations. Different values of color distances are formed from different images. After finding color distance, Euclidean distance is formed by finding difference between

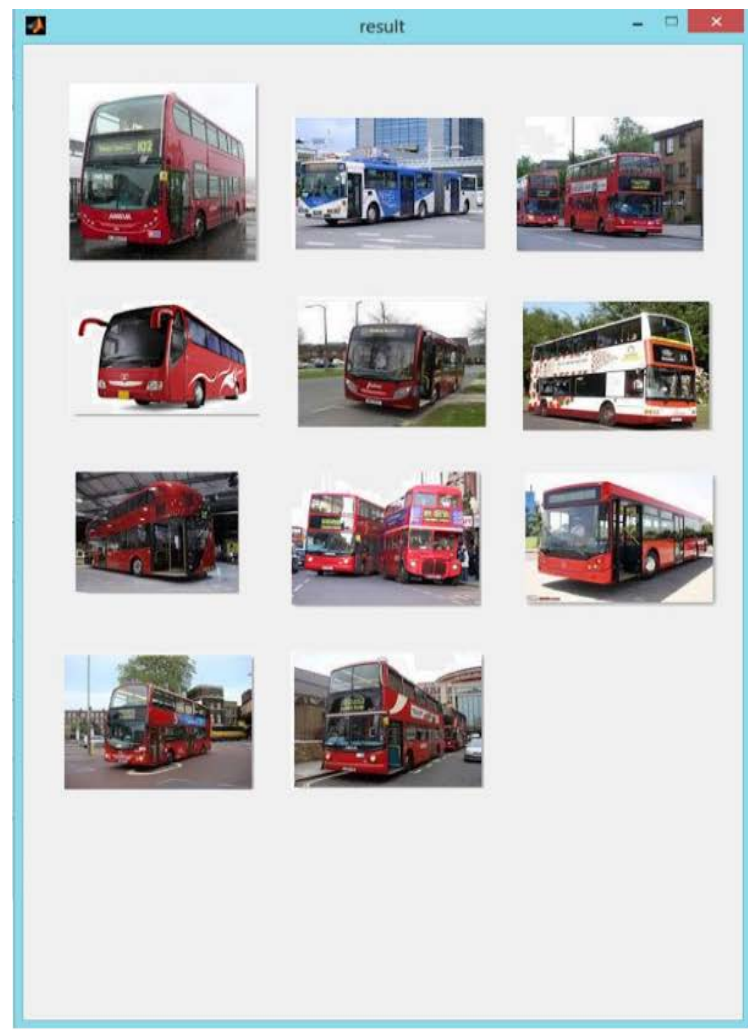

(a) query image and retrieved image. After this, a similar formula is applied on Bus image and Horse image as shown in Figure 6.

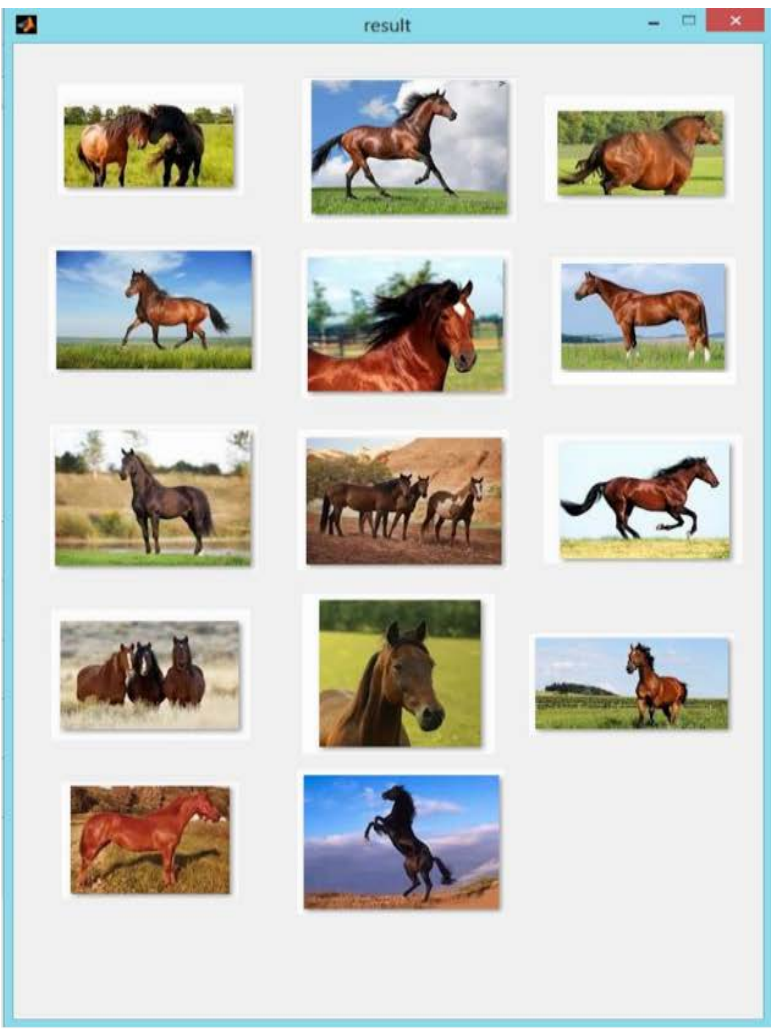

Fig. 6Search results (a) image 200.jpg (b) image 298.jpg

(h)

\section{V.CONCLUSION}

The application uses color correlogram and pyramid structured $(\mathrm{T})$ wavelet transform for indexing the images and retrieve them from image database. Color distance is calculated using quadratic equationand

texture is calculated using Euclidean difference between the query and retrieved image. In future, more emphasis should be on improving the design of content based features of an image. In our paper we have only done color analysis, texture analysis and analysis of information about object location where the shape is discarded. Thus, this paper shows the retrieval of an image by using the above mentioned methods. Some of images are not be similar even though they share similar color distribution and texture in some results. In future a system that contains information about image location and its shape can be developed for improved retrieval.

\section{REFERENCES}

[1] S.M. Singh and K. Hemachandran, "Image retrieval based on the combination of color histogram and color moment."International Journal of Computer Applications, Vol. 58, No. 3, pp. 27-34, 2012.
[2] B.Dinakaran, J.Annapurna, and C.A.Kumar, "Interactive image retrieval using text and image conten," Cybern Inf Tech, Vol. 10,pp. 20-30, 2010.

[3] H.B. Kekre and D.Mishra, "Sectorization of Full Kekre's Wavelet Transform for Feature extraction of Color Images,"International Journal of Advanced Computer Science and Applications, Vol. 2, No. 2, pp. 58-68, 2011.

[4] S.V. Sakhare and V.G.Nasre, "Design of feature extraction in content based image retrieval (CBIR) using color and texture," International Journal of Computer Science \& Informatics, Vol. 1, No. 2, pp. 57-61, 2011.

[5] E.Sirisha, P.Sanoop, P.H. Vishnu and Y.Srinivas, "Image Retrieval using Wavelet Decomposition Color Correlogram and Color Mean,"International Journal of Advanced Research in Computer Science and Software Engineering,Vol. 3, No. 9, pp. 1176-1180, 2013.

[6] F.Long, H.Zhang and D.D.Feng, "Fundamentals of content-based image retrieval. In Multimedia Information Retrieval and Management, Springer, Berlin, Heidelberg,pp. 1-26, 2003.

[7] K.Haridas and A.S.Thanamani, "Well-organized content based image retrieval system in RGB Color Histogram, Tamura Texture and Gabor Feature," International Journal of Advanced Research in Computer and Communication Engineering,Vol. 3, No. 10, pp. 82428248,2014.

[8] M.Danish, R.Rawat and R.Sharma, "A survey: content based image retrieval based on color, texture, shape \& neuro fuzzy,"International Journal of Engineering Research and Applications, Vol. 3, No. 5, pp. 839-844, 2013. 
[9] M. Lux and S.A.Chatzichristofis, "Lire: lucene image retrieval: an extensible java cbir library", In Proceedings of the 16th ACM international conference on Multimedia,pp. 1085-1088, 2008.

[10] P.A. Deole and Longadge, "Content based image retrieval using color feature extraction with KNN classification,"International Journal of Computer Science and Mobile Computing, Vol. 3, No. 5, pp. 1274-1280, 2014.

[11] S.M.H.Khan, A. Hussain and I.F.T.Alshaikhli, Comparative study on content-based image retrieval (CBIR). in International Conference onAdvanced Computer Science Applications and Technologies-2012, IEEE.pp. 61-66, 2012.

[12] M.J.Swain and D.H.Ballard, "Color indexing, International Journal of Computer Vision,”Vol. 7, No. 1, pp. 11-32,1991.

[13] B. Ramamurthy and K.R.Chandran, "Content based medical image retrieval with texture content using gray level co-occurrence matrix and k-means clustering algorithms,"Journal of Computer Science,Vol. 8, No. 7, pp. 1070-1076, 2012.

[14] N.Jain and D.S.Salankar, "Color \& texture feature extraction for content based image retrieval,"IOSR Journal of Electrical and Electronics Engineering (IOSR-JEEE),pp. 2278-1676, 2014.

[15] P.RaviTheja and S.Balaji, "A novel approach of an effective image retrieval scheme using color, texture and shape features," International Journal of Computer \& Organization Trends,Vol. 3, No. 2, pp. 9-13, 2013.

[16] R.Bala and R.Sharma, "Efficient Method of CBIR Using Combination of Shape, Color and Texture Feature with Fuzzy Technique,"International Journal of Advanced Research in Electronics and Communication Engineering, Vol. 3, No. 8, pp. 909913, 2014.
[17] A.R. Kumar and D.Saravanan, "Content based image retrieval using color histogram,"International journal of computer science and information technologies, Vol. 4, No. 2, pp. 242-245, 2013.

[18] S.Vidivelli and S.S.Devi,"Wavelet based integrated color image retrieval,"inInternational Conference on Recent Trends in Information Technology, IEEE,pp. 853-856, 2011.

[19] A.Kaur, V.K.Banga and N.Kaur, "Color and texture based image retrieval: a proposed method,"International Journal of Research in Engineering and Technology,Vol. 2, No. 1, pp. 498-501, $2013 .$.

[20] M.S.R. Janani and P.Sebhakumar, "An improved cbir method using color and texture properties with relevance feedback",International Journal of Innovative Research in Computer and Communication Engineering,Vol. 2, No. 1, pp. 47-54, 2014.

[21] P.Jayaprabha and R.Somasundaram, "Content based image retrieval methods using graphical image retrieval algorithm (GIRA),"International Journal of Information and Communication Technology Research,Vol. 2, No. 1, pp. 9-14, 2012.

[22] A. Oberoi and M.Singh, "Content based image retrieval system for medical databases (CBIR-MD)-lucratively tested on endoscopy, dental and skull images,"International Journal of Computer Science Issues, Vol. 9, No. 3, pp. 300-306, 2012.

[23] J.Huang, , S.R.Kumar, M.Mitra, W.J. Zhu and R.Zabih, "Image indexing using color correlograms. In Proceedings on Computer Vision and Pattern Recognition-1997, IEEE Computer Society, pp. 762-768, 1997.

[24] D.John and S.T.Tharani, "Content based image retrieval using HSVcolor histogram and GLCM," International Journal of Advance Research in Computer Science and Management Studies, Vol. 2, No. 1, pp. 246-253,2014. 